\title{
STMN1 accumulation is associated with dysplastic and neoplastic lesions in patients with ulcerative colitis
}

\author{
KATSUYA OSONE $^{1}$, TAKEHIKO YOKOBORI ${ }^{1-3}$, CHIKA KATAYAMA ${ }^{1}$, RYO TAKAHASHI ${ }^{1}$, \\ RYUJI KATO $^{1}$, HIRONORI TATSUSKI ${ }^{1}$, TAKAHIRO TAKADA ${ }^{1}$, REINA YAJIMA ${ }^{1}$, \\ YOKO MOTEGI $^{1}$, HIROOMI OGAWA ${ }^{1}$, TAKAAKI FUJII ${ }^{1}$, HITOSHI OJIMA ${ }^{4}$, \\ JUNICHI NAKAMURA ${ }^{5}$, TAKASHI YAO $^{6}$, KEN SHIRABE $^{1,7}$ and HIROYUKI KUWANO ${ }^{1}$
}

\begin{abstract}
${ }^{1}$ Department of General Surgical Science, Gunma University, Graduate School of Medicine; ${ }^{2}$ Department of Innovative Cancer Immunotherapy, Gunma University; ${ }^{3}$ Gunma University Initiative for Advanced Research (GIAR), Maebashi, Gunma 371-8511; ${ }^{4}$ Department of Gastroenterological Surgery, Gunma Prefectural Cancer Center, Ohta, Gunma 373-8550; ${ }^{5}$ Department of Gastroenterological Surgery, Saitama Red Cross Hospital, Ohmiya, Saitama 330-8553; ${ }^{6}$ Department of Human Pathology, Juntendo University Graduate School of Medicine, Bunkyouku, Tokyo 113-8421; ${ }^{7}$ Department of Hepatobiliary and Pancreatic Surgery, Gunma

University Graduate School of Medicine, Maebashi, Gunma 371-8511, Japan
\end{abstract}

Received January 1, 2019; Accepted April 23, 2019

DOI: $10.3892 / 01.2019 .10814$

\begin{abstract}
Ulcerative colitis (UC) is thought to be associated with precancerous lesions that can ultimately lead to colon cancer. Therefore, diagnostic markers for colorectal dysplasia and cancer are urgently needed for patients with UC. Stathmin 1 (STMN1) is a novel cancer biomarker that is also a novel target for treatment in several cancers, including colon cancer. However, few studies have investigated the relationship between STMN1 expression and clinical features in colorectal dysplasia and cancer in patients with UC. The present study examined the clinical significance of STMN1 expression in colorectal dysplasia and cancer with UC. The present study performed an immunohistochemical analysis of 31 clinical colorectal samples from eight patients with colorectal dysplasia and/or cancer to assess the relationships between STMN1 expression and clinicopathological features including mismatch repair protein expression, rate of Ki-67 positivity, differentiation level, TNM stage, and UC duration. STNM1 expression was detected in $95.7 \%$ of dysplastic and cancerous lesions, whereas p53, the current diagnostic marker, was not expressed in $39.1 \%$ of dysplastic and cancerous lesions. Furthermore, STMN1 expression was associated with a high rate of positivity for Ki-67, a
\end{abstract}

Correspondence to: Dr Takehiko Yokobori, Department of Innovative Cancer Immunotherapy, Gunma University, 3-39-22 Showa-machi, Maebashi, Gunma 371-8511 Japan

E-mail: bori45@gunma-u.ac.jp

Key words: colitic cancer, inflammatory bowel disease, ulcerative colitis, carcinogenesis, dysplasia proliferation marker. Our data suggest that STMN1 in the colonic mucosa of UC patients may be useful as an early diagnostic marker of dysplasia and colitic cancer.

\section{Introduction}

The inflamed colonic mucosa of patients with ulcerative colitis (UC), an inflammatory disease affecting the colorectal region, is suggested to be a site for the development of precancerous lesions that can ultimately progress to colon cancer (1). As colorectal dysplasia and cancer is an important complication of UC, surveillance endoscopy is recommended for the evaluation of inflammation and cancer in patients who have had UC for more than eight years (2). Total colectomy is considered for patients diagnosed with high-grade colorectal dysplasia or cancer during surveillance. It is recommended that the frequency of surveillance be increased in cases of low-grade dysplasia. However, due to the underlying chronic inflammation in the colonic mucosa of patients with UC, histological assessments of UC lesions for carcinomatous changes are often challenging (3). Therefore, diagnostic markers that can identify colorectal dysplasia and cancer in the presence of long-standing colorectal inflammation in UC are sorely needed.

Recently, the expression levels of p53, Ki-67, and chromogranin A have been reported to be useful for the diagnosis of colorectal dysplasia and cancer in UC patients (4-6). Although mismatch repair deficiency (MMRD) is reportedly associated with carcinogenic processes in the colon, the significance of MMRD in colitic cancer remains controversial $(7,8)$. Additionally, p53 expression has been used primarily as an early diagnostic marker for colorectal dysplasia and cancer. However, false positive results due to chronic inflammation and false negative results despite p53 mutations are critical issues in the diagnostic evaluation of patients with UC. Therefore, development of highly sensitive and specific 
diagnostic markers is needed for improved surveillance in this patient population.

Stathmin 1 (STMN1) is a major cytosolic phosphoprotein that regulates microtubule dynamics by promoting microtubule destabilization (9). Intriguingly, increased STMN1 expression has been observed in numerous cancers including colorectal cancer, and cancer patients with increased STMN1 expression were reported to exhibit aggressive tumor phenotypes and poor prognosis (10). Therefore, STMN1 is considered potentially useful not only as a cancer biomarker but also as a novel target for cancer treatment. However, few studies have investigated the relationship between STMN1 expression levels and clinical features in colorectal dysplasia and cancer in UC patients.

The purpose of the present study was to determine the clinical significance of STMN1 in colorectal dysplasia and cancer due to UC. We performed immunohistochemical analysis on 31 clinical colorectal samples from eight patients with colorectal dysplasia and/or cancer. Our results demonstrate the presence of a relationship between STMN1 expression and several clinicopathological features, including MMRD status, rate of Ki-67 positivity, differentiation level, TNM grade, and UC duration.

\section{Materials and methods}

Patients and samples. Eight patients (six males and two females) with UC who underwent surgical resection in Gunma University Hospital, Saitama Red Cross Hospital, and Gunma Prefectural Cancer Center between 1999 and 2014 were included in this retrospective study. The median age of the patients was 59 years (range 37-76 years). One patient had only dysplasia. Some patients had two or more tumors, and all dysplastic and cancerous lesions were evaluated. All dysplastic lesion samples were only obtained from patients with high-grade dysplasia; patients with low-grade dysplasia were not included in the study. This study conformed to the tenets of the Declaration of Helsinki and was approved by the Institutional Review Board for Clinical Research at the Gunma University Hospital (Maebashi, Gunma, Japan). Patient consent was obtained via the opt-out method. Table I summarizes patient information. For accurate pathological diagnosis of dysplastic lesions in UC patients, all dysplasia and cancer tumor sections were evaluated by a specialized pathologist, Dr. Yao T (Department of Human Pathology, Juntendo University Graduate School of Medicine).

Immunohistochemical staining. Paraffin-embedded blocks of all surgical resection specimens obtained from UC patients were cut into sections $4 \mu \mathrm{m}$ in thickness and mounted on glass slides. Sections were deparaffinized using xylene and dehydrated in alcohol. Endogenous peroxidase was inhibited using $0.3 \% \mathrm{H}_{2} \mathrm{O}_{2} /$ methanol for $30 \mathrm{~min}$ at room temperature. Then, the sections were soaked in heated water supplemented with $0.5 \%$ Immunosaver (Nishin EM, Tokyo, Japan) at $98^{\circ} \mathrm{C}$ for $45 \mathrm{~min}$. Nonspecific antigens were blocked by serum-free Protein Block (DAKO, Glostrup, Denmark) at room temperature for $30 \mathrm{~min}$. Next, the sections were incubated with primary antibodies against STMN1 (mouse monoclonal, 1:200; Santa Cruz Biotechnology, Santa Cruz, CA, USA), p53
Table I. Clinical characteristics of the patients with UC in the present study.

\begin{tabular}{lc}
\hline Characteristics & Patient number $(\mathrm{n}=8)$ \\
\hline Age (years) & $59(37-76)$ \\
Median (range) & $6 / 2$ \\
$<70 / \geq 70$ & \\
Sex & $6 / 2$ \\
Male/Female & \\
Number of sample & $8 / 12 / 11$ \\
Normal/Dysplasia/Cancer & \\
Differentiation & $2 / 5 / 4$ \\
Poor/Moderate/Well & \\
Stage & $2 / 1$ \\
I/II & $0 / 2$ \\
IIIA/IIIB & $1 / 1$ \\
IIIC/IV & \\
Duration of disease (years) & $15.3(4-27)$ \\
Median (range) &
\end{tabular}

UC, ulcerative colitis.

(mouse monoclonal anti-human [DO-7], 1:100; DAKO), Ki67 (mouse monoclonal anti-human [MIB-1], 1:300; DAKO), MLH1 (mouse monoclonal anti-human [ES05]; DAKO), MSH2 (mouse monoclonal anti-human [FE11]; DAKO), MSH6 (rabbit monoclonal anti-human [EP49]; DAKO), and PMS2 (rabbit monoclonal anti-human [EP51]; DAKO) at $4^{\circ} \mathrm{C}$ for $24 \mathrm{~h}$. After washing with phosphate-buffered saline, the sections were incubated in Histofine Simple Stain ${ }^{\mathrm{TM}}$ MAX PO (MULTI) solution (Nichirei, Tokyo, Japan) for $45 \mathrm{~min}$ to visualize primary antibodies. The chromogen 3,3'-diaminobenzidine tetrahydrochloride was applied as a $0.02 \%$ solution, which contained $0.005 \% \mathrm{H}_{2} \mathrm{O}_{2}$ in $50 \mathrm{mM}$ ammonium acetate-citrate acid buffer ( $\mathrm{pH}$ 6.0). Finally, nuclear counterstaining was performed using Mayer's hematoxylin solution. Negative controls for immunohistochemical staining involved replacing primary antibodies with phosphate-buffered saline in $0.1 \%$ bovine serum albumin and confirming a lack of staining.

Assessment of STMN1, p53, Ki-67, and mismatch repair protein expression. We evaluated cytoplasmic staining for STMN1 in noncancerous tissues as well as dysplastic and cancerous tissues from patients with UC. Cytoplasmic STMN1 was scored as follows: 0 , no staining; $1+, 1-10 \%$ staining; $2+$, $11-50 \%$ staining; and $3+, 51-100 \%$ staining. The optimal cutoff point was defined as follows: Grades 0 and 1 were considered negative, and grades 2 and 3 were considered positive. p53-positive cells were defined as those with a brown-stained nucleus, regardless of staining intensity. The following four staining patterns were identified: Positive cells in most of the lesion (diffuse); positive cells aggregated in a focal area of the lesion (nested); small numbers of isolated positive cells scattered throughout the lesion (scattered); and negative. Positive 
Non-cancerous
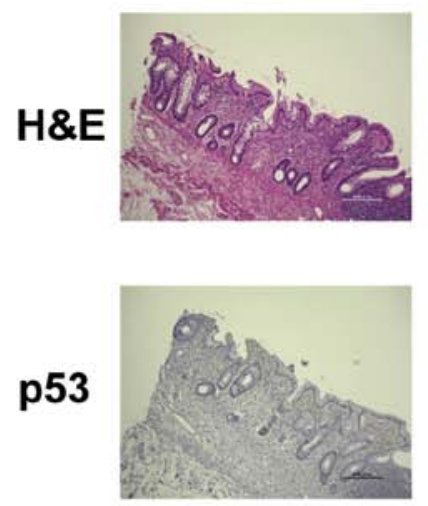

$(-)$

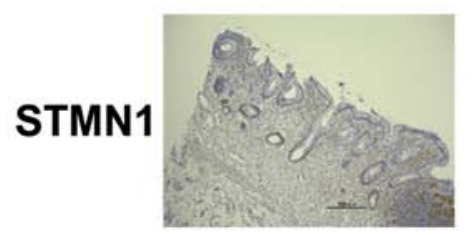

$(-)$
Dysplasia
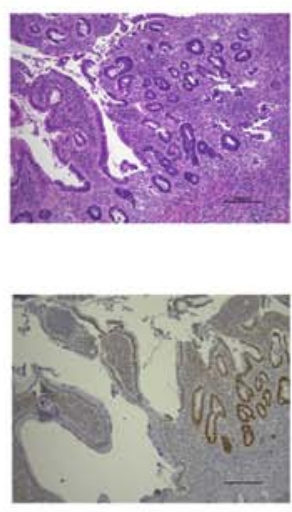

$(+++)$

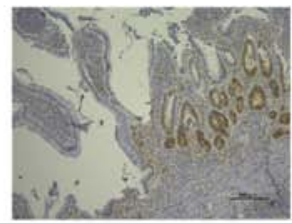

$(+++)$
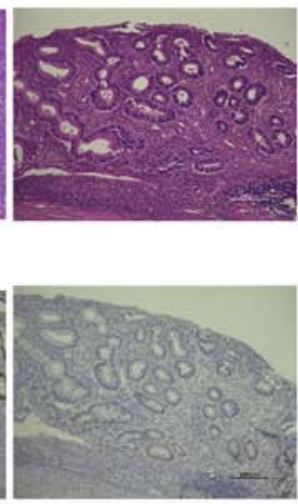

$(-)$

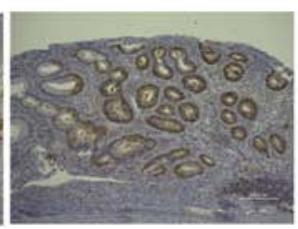

$(+++)$
Colitic cancer
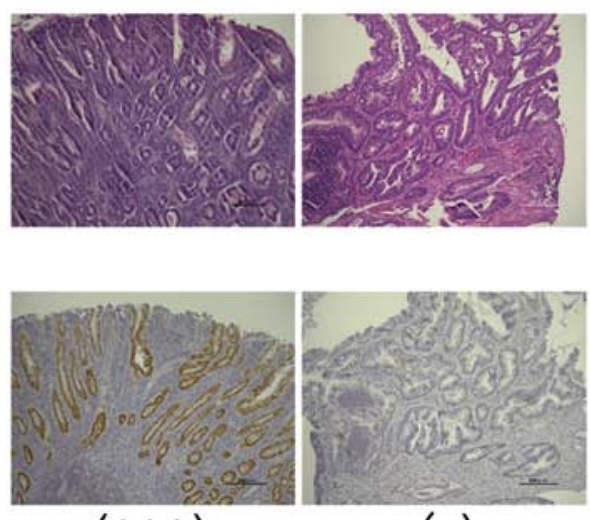

$(+++)$

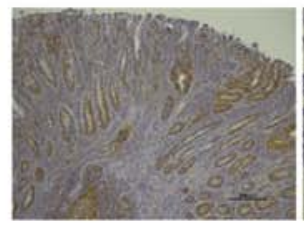

$(+++)$

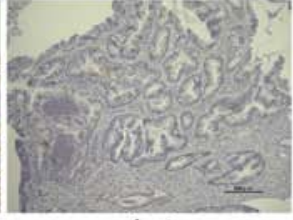

$(-)$

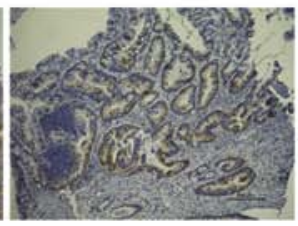

$(+++)$

Figure 1. Expression of STMN1 and p53 in the colitic mucosa of patients with UC and colitic cancer. Upper panel: Representative images of H\&E staining in samples of noncancerous tissues as well as dysplastic and cancerous lesions. Middle panel: p53 expression in samples of noncancerous tissues as well as dysplastic and cancerous lesions. Lower panel: STMN1 expression in samples of noncancerous tissues as well as dysplastic and cancerous lesions. UC, ulcerative colitis; STMN1, stathmin 1; H\&E, hematoxylin \& eosin.

p53 protein expression was defined as either a diffuse or nested pattern, whereas negative $\mathrm{p} 53$ protein expression was defined as a scattered pattern throughout the lesion or negative staining, as described previously (6). The Ki-67 labeling index was scored as a percentage of positively stained cells. MMRD was defined as the complete absence of the expression of at least one MMR protein (MLH1, MSH2, MSH6, or PMS2).

Statistical analysis. The JMP software package (SAS Institute Inc., Cary, NC, USA) was used to perform all statistical analyses. Chi-square tests were used to analyze associations between STMN1 and p53 expression levels. Wilcoxon's test was used to analyze associations between STMN1 expression and the rate of Ki-67 positivity. All differences were considered statistically significant at $\mathrm{P}<0.05$.

\section{Results}

Immunohistochemical analysis of STMN1 expression in colorectal tissue specimens of patients with UC. As STMN1 was expressed in the cytoplasm of colorectal tissue sections obtained from patients with UCs, cytoplasmic STMN1 expression was evaluated in sections of noncancerous tissues as well as dysplastic and cancerous lesions from eight patients with UC. STMN1 was highly expressed in the cytoplasm of dysplastic as well as cancerous tissue sections, whereas cytoplasmic STMN1 staining could not be detected in noncancerous tissue sections (Fig. 1). In contrast, p53 expression, which was identified previously as a clinical diagnostic marker for colorectal dysplasia and cancer, was absent in the dysplastic and cancerous sections of some UC patients in the present study. Importantly, these p53-negative sections were positive for STMN1, based on immunohistochemical analysis (Fig. 1).

Significance of the expression levels of STMN1, p53, Ki-67, and MMR proteins in the colonic mucosa of patients with $U C$. Next, we analyzed the relationships among STMN1 and p53 expression levels, Ki-67 labeling index, MMRD, level of differentiation, TNM stage, and duration of disease in 31 colitic mucosa samples from eight patients with UC. Table II summarizes clinicopathological characteristics and results of immunohistochemical analysis of these samples. In the study cohort, STMN1 was highly expressed in dysplastic as well as cancerous specimens, whereas noncancerous tissues were not positive for STMN1 based on immunohistochemical staining (Fig. 2). The rate of STMN1 positivity in 12 dysplastic and 11 colitic cancer tissue sections was 91.7 and $100 \%$, respectively (Fig. 2; Table II). The rate of p53 positivity in the dysplastic and cancerous tissue sections were 58.3 and $63.6 \%$, respectively. Conversely, the rate of p53 positivity in the noncancerous tissue sections was $12.5 \%$. The rate of positive STNM1 staining in the cancerous tissue sections was higher than that of p53 (Fig. 2; P=0.001), and that the rate of positive STNM1 staining in the dysplastic and cancerous tissue sections was higher than that of p53 $(95.7 \%$ vs $60.9 \%$; Fig. $2 ; \mathrm{P}=0.003$ ). The positive predictive, negative predictive, and AUC values of STMN1 in the dysplastic and cancerous tissue sections were defined as $100,83.3 \%$, and 0.97826 , respectively. These values were higher than those of p53, which were $93,47.4 \%$, and 0.73261 respectively. 
Table II. Clinicopathological characteristics in ulcerative colitis patients with high-grade dysplasia and colitic cancer.

\begin{tabular}{|c|c|c|c|c|c|c|c|c|c|c|}
\hline $\begin{array}{l}\text { Pathological } \\
\text { diagnosis }\end{array}$ & Location & p53 & STMN1 & MMRD & $\begin{array}{c}\mathrm{Ki}-67 \\
\text { positive } \\
\text { rate }(\%)\end{array}$ & Differentiation & $\begin{array}{c}\mathrm{T} \\
\text { factor }\end{array}$ & $\mathrm{N}$ factor & $\mathrm{M}$ factor & Stage \\
\hline \multicolumn{11}{|l|}{ Case 1} \\
\hline Normal & $\mathrm{Ce}$ & + & - & - & 3.0 & - & - & $1 \mathrm{a}$ & Negative & IIIB \\
\hline Dys & $\mathrm{Ce}$ & + & + & - & 10.2 & & & & & \\
\hline Dys & $\mathrm{Ce}$ & - & + & - & 3.8 & & & & & \\
\hline Dys & $\mathrm{Ce}$ & - & + & - & 3.5 & & & & & \\
\hline Dys & A & + & - & - & 18.6 & & & & & \\
\hline Dys & $\mathrm{S}$ & + & + & - & 5.4 & & & & & \\
\hline Dys & $\mathrm{S}$ & + & + & - & 4.7 & & & & & \\
\hline $\mathrm{Ca}$ & $\mathrm{Ce}$ & - & + & - & 28.0 & Moderate & 3 & & & \\
\hline $\mathrm{Ca}$ & A & + & + & - & 10.0 & Moderate & $4 a$ & & & \\
\hline $\mathrm{Ca}$ & $\mathrm{S}$ & + & + & - & 44.6 & Moderate & 2 & & & \\
\hline \multicolumn{11}{|l|}{ Case 2} \\
\hline Normal & $\mathrm{D}$ & - & - & - & 24.0 & - & - & $2 \mathrm{a}$ & Negative & IIIC \\
\hline Dys & $\mathrm{D}$ & - & + & - & 27.0 & - & - & & & \\
\hline $\mathrm{Ca}$ & A & + & + & + & 24.6 & Poor & $4 a$ & & & \\
\hline $\mathrm{Ca}$ & $\mathrm{D}$ & + & + & - & 63.0 & Moderate & 1 & & & \\
\hline \multicolumn{11}{|l|}{ Case 3} \\
\hline Normal & $\mathrm{T}$ & - & - & - & 46.6 & - & - & $1 \mathrm{a}$ & Negative & IIIB \\
\hline Dys & $\mathrm{T}$ & + & + & - & 39.0 & & & & & \\
\hline Dys & $\mathrm{D}$ & + & + & - & 49.0 & & & & & \\
\hline $\mathrm{Ca}$ & $\mathrm{T}$ & + & + & - & 68.8 & Moderate & 3 & & & \\
\hline \multicolumn{11}{|l|}{ Case 4} \\
\hline Normal & $\mathrm{S}$ & - & - & - & 1.8 & - & - & $2 \mathrm{a}$ & M1b & IVB \\
\hline Dys & $\mathrm{S}$ & - & + & - & 51.0 & & & & & \\
\hline $\mathrm{Ca}$ & $\mathrm{S}$ & - & + & - & 63.2 & Well & $4 a$ & & & \\
\hline \multicolumn{11}{|l|}{ Case 5} \\
\hline Normal & $\mathrm{S}$ & - & - & - & 16.6 & - & - & 0 & Negative & I \\
\hline Dys & $\mathrm{S}$ & - & + & - & 73.4 & & & & & \\
\hline $\mathrm{Ca}$ & $\mathrm{S}$ & + & + & - & 39.8 & Well & 2 & & & \\
\hline \multicolumn{11}{|l|}{ Case 6} \\
\hline Normal & $\mathrm{S}$ & - & - & - & 5.6 & - & - & 0 & Negative & IIC \\
\hline $\mathrm{Ca}$ & $\mathrm{S}$ & - & + & - & 26.6 & Poor & $4 b$ & & & \\
\hline \multicolumn{11}{|l|}{ Case 7} \\
\hline Normal & $\mathrm{T}$ & - & - & - & 40.0 & - & - & - & - & - \\
\hline Dys & $\mathrm{RS}$ & + & + & - & 86.2 & & & & & \\
\hline \multicolumn{11}{|l|}{ Case 8} \\
\hline Normal & $\mathrm{Rb}$ & - & - & - & 1.4 & - & - & 0 & Negative & I \\
\hline $\mathrm{Ca}$ & $\mathrm{D}$ & - & + & - & 49.8 & Well & 1 & & & \\
\hline $\mathrm{Ca}$ & $\mathrm{Rb}$ & - & + & - & 12.2 & Well & 2 & & & \\
\hline
\end{tabular}

MMRD, mismatch repair deficiency; Dys, dysplastic specimen; Ca, cancerous specimen; STMN1, stathmin 1; Ce, cecum; A, ascending colon; $\mathrm{T}$, transverse colon; D, descending colon; S, sigmoid colon; RS, rectosigmoid; Rb, lower rectum.

Furthermore, the median Ki-67 labeling index in sections with high STMN1 expression $(33.5 \%, 22 / 31)$ was higher than that in sections with low STMN1 expression $(16.6 \%, 9 / 31$; Fig. 3; $\mathrm{P}=0.04)$. Only one colitic cancer sample was identified as mismatch repair-deficient in this study (Table II).

\section{Discussion}

In the present study, we observed that $95.7 \%$ of sections of dysplastic and cancerous tissues from all patients with UC were positive for STMN1 staining. In contrast, p53, which is 


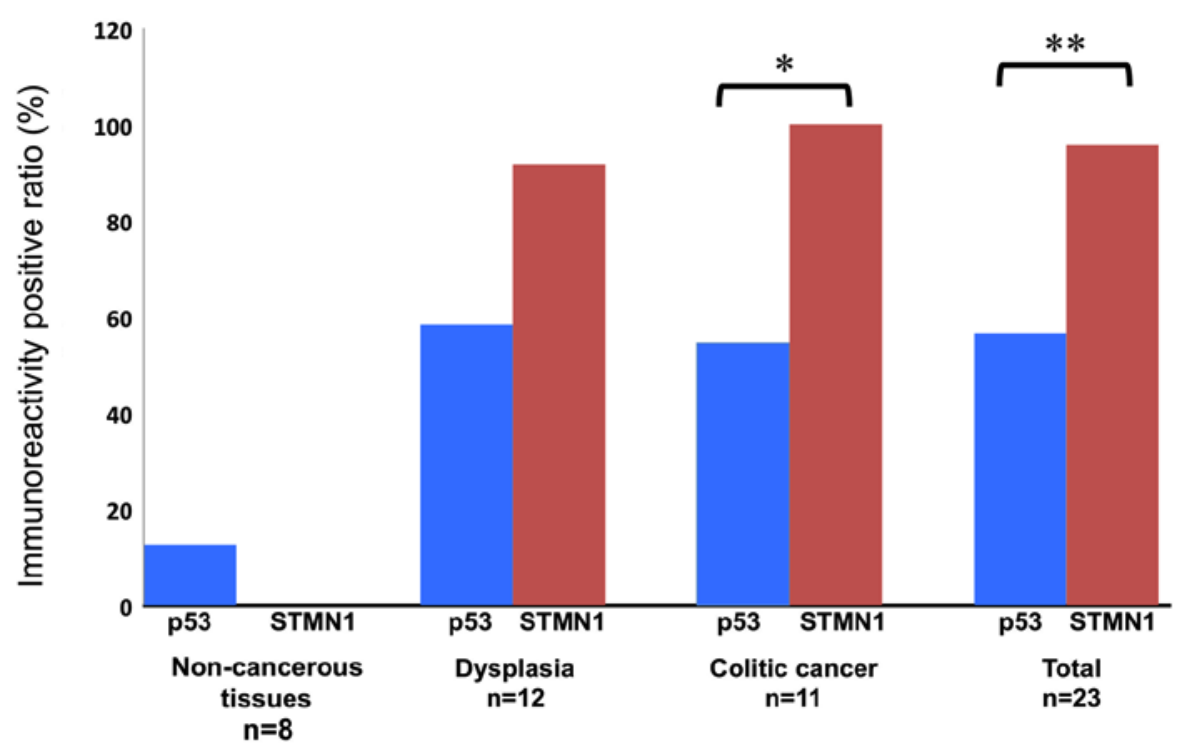

Figure 2. Rate of positivity for STMN1 and p53 staining in samples from eight noncancerous tissues from eight patients, twelve dysplastic lesions from six patients, and eleven colitic cancer lesions from seven patients among a total of eight patients with ulcerative colitis. ${ }^{*} \mathrm{P}=0.011$ and ${ }^{* * *} \mathrm{P}=0.003$, as indicated. STMN1, stathmin 1.

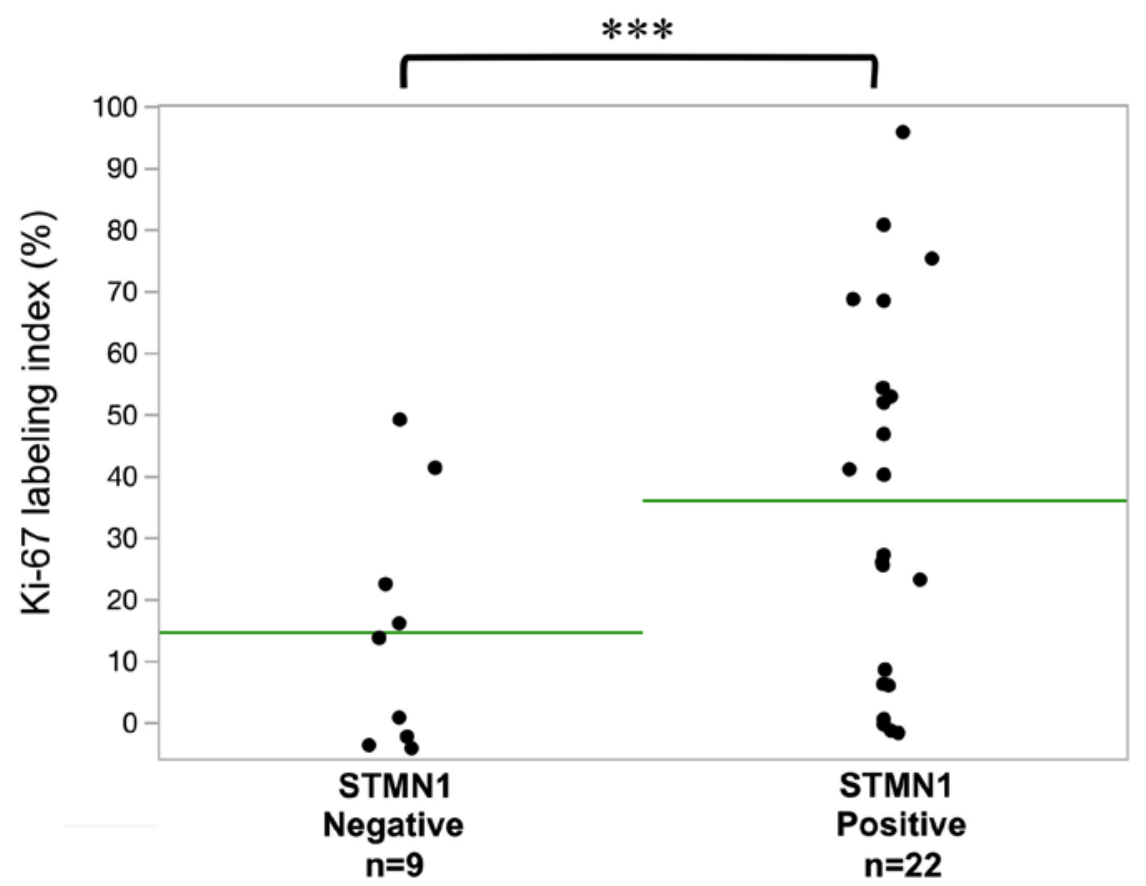

Figure 3. Relationship between Ki-67 labeling index and STMN1 expression in 31 colitic mucosa samples obtained from eight patients with ulcerative colitis. The Ki-67 labeling index among samples with high STMN1 expression $(\mathrm{n}=22)$ was higher than that of samples with low STMN1 expression $\left(\mathrm{n}=9\right.$; ${ }^{* * *} \mathrm{P}=0.04$, as indicated). Horizontal lines indicate mean values for each group. STMN1, stathmin 1.

the currently used diagnostic marker for colorectal dysplasia and cancer, was expressed in only $60.9 \%$ of the sections of dysplastic and cancerous lesions. Moreover, STMN1 expression observed in the colonic mucosa as well as the dysplastic and cancerous lesions of eight patients with UC in the current study was associated with a high rate of Ki-67 positivity, a marker of proliferation.

Our data were consistent with previous studies reporting the rate of $\mathrm{p} 53$ positivity as $45.0-77.8 \%$ in dysplastic lesions and $57.0-90.9 \%$ in colitic cancer among patients with UC $(4-6,11)$.
Additionally, in the current study, STMN1 expression was detected in $95.7 \%$ of sections of dysplasia and cancerous lesions; further, STMN1 expression was not detected in any section of noncancerous lesions. These observations suggest that STMN1 expression might be more accurate and useful than the current diagnostic marker, p53, for the diagnosis of dysplasia and cancer in patients with UC.

Colitic cancer secondary to chronic inflammation due to UC presents a clinically significant problem (12), and total colonoscopy is often used for the surveillance and diagnosis of 
colitic cancer in affected patients. However, less invasive and low-cost clinical modalities are needed to avoid invasive colonoscopy procedures, which are associated with high medical costs relative to laboratory tests in general. Elevated STMN1 levels in serum and urine samples from patients with bladder cancer support its utility as a cancer biomarker (13). Future studies are necessary to assess whether elevated STMN1 expression in liquid samples from patients with UC can be utilized as a new marker to predict the presence of dysplasia and colitic cancer.

p53 staining patterns are generally classified as diffuse, sporadic, scattered, or nested. Because the region observable in an endoscopic biopsy is limited, assessments of biopsy samples from patients with UC based on p53 staining might overlook colitic cancer and dysplasia using this classification. In the current study, we observed that STMN1 stained uniformly within entire cancerous lesions, further providing support for STMN1 staining of biopsy samples as a useful marker of dysplasia and colitic cancer in UC.

Previously, it was reported that $67 \%$ of colitic cancer samples showed high-level MSI (microsatellite instability) phenotypes as MMR protein deficiency (7). However, $9.1 \%$ of colitic cancer samples were reported to show MMR deficiency (8). The MMR frequency in colitic cancer was different in each report and the association of MMR with colitic cancer is controversial. In the present study, we evaluated for the first time the association between expression of STMN1 and MMR deficiency; however, we detected MMR deficiency in only one sample and we were unable to perform sufficient statistical analysis using our limited data. In the future, multi-centric large cohort analyses will be required to clarify the relationship between STMN1 and MMR deficiency, although sample collection will present difficulties as colitic cancer is rare.

Zhang et al (10) reported previously that the rate of STMN1 positivity was $62.9 \%$ in colon cancer samples from non-UC patients. However, this rate in the present study was $100 \%$. In contrast to colon cancers in non-UC patients, colitic cancer has been reported to be in accordance with the dysplasia-carcinoma sequence hypothesis, which is associated with a higher p53 mutation rate in dysplasia and colitic cancer in UC patients than that in non-UC patients (14). Interestingly, mutant p53 was reported to induce STMN1 expression (15). Therefore, it was suggested that the difference in p53 mutation rate between colitic cancer and sporadic colon cancer influences the high rate of STMN1 positivity in colitic cancers.

This study had several limitations. First, the limited number of subjects in this study may have contributed to less detection power in this study. Second, we did not compare the expression significance of STMN1 in colon cancer from UC patients with that from non-UC patients using the same immunohistochemical method. Third, we did not implement any functional studies on the relationship between colon cancer-related genes, including STMN1 and colon cancer, using cell lines derived from colitic cancer patients.

Elevated STMN1 expression was observed in dysplastic lesions from patients with UC. Our data suggest that STMN1 expression in the colonic mucosa of patients with UC might be useful as an early diagnostic marker of dysplasia and colitic cancer.

\section{Acknowledgements}

The authors would like to thank Ms. Yukie Saito, Ms. Tomoko Yano, Ms. Yuka Matsui, Ms. Sayaka Okada, and Ms. Kayoko Takahashi (Department of General Surgical Science, Gunma University, Graduate School of Medicine) for their assistance.

\section{Funding}

The present study was supported by JSS Young Researcher Award from Japan Surgical Society, Gunma University Clinical Biobank, and Grants-in-Aid for Scientific Research from the Japan Society for the Promotion of Science (JSPS) (grant nos. JP 26461969, JP15K10129, JP15K10085, JP26350557 and $17 \mathrm{~K} 19893)$.

\section{Availability of data and materials}

The datasets used or analyzed during the present study are available from the corresponding author on reasonable request.

\section{Authors' contributions}

The present study was designed and organized by HK, KS, TYo and KO. Clinical samples and data were collected by HOg, RY, YM, TT, HT, RK, RT, CK, JN, HOj and KO. Immunostaining was evaluated by TF, TYa and KO. HK, TYo, and KO contributed to data analysis, interpretation and drafting manuscript. All authors read and approved the final manuscript.

\section{Ethics approval and consent to participate}

The present study was approved by the institutional guidelines of Gunma University Graduate School of Medicine (Maebashi, Japan). Written informed concept was obtained by the patients who agreed with the future research using resected samples prior to surgery. Therefore, the opt-out method for the STMN1 project was performed using archived samples.

\section{Patient consent for publication}

Patient agreement was obtained via the opt-out method.

\section{Competing interests}

The authors declare that they have no competing interests.

\section{References}

1. Morson BC: Cancer in ulcerative colitis. Gut 7: 425-426, 1966.

2. Van Assche G, Dignass A, Bokemeyer B, Danese S, Gionchetti P, Moser G, Beaugerie L, Gomollón F, Häuser W, Herrlinger K, et al: Second European evidence-based consensus on the diagnosis and management of ulcerative colitis part 3: Special situations. J Crohns Colitis 7: 1-33, 2013.

3. Eaden J, Abrams K, McKay H, Denley H and Mayberry J: Inter-observer variation between general and specialist gastrointestinal pathologists when grading dysplasia in ulcerative colitis. J Pathol 194: 152-157, 2001.

4. Kobayashi K, Tomita H, Shimizu M, Tanaka T, Suzui N, Miyazaki T and Hara A: p53 Expression as a diagnostic biomarker in ulcerative colitis-associated cancer. Int J Mol Sci 18: E1284, 2017. 
5. Wong NA, Mayer NJ, MacKell S, Gilmour HM and Harrison DJ: Immunohistochemical assessment of Ki67 and p53 expression assists the diagnosis and grading of ulcerative colitis-related dysplasia. Histopathology 37: 108-114, 2000.

6. Shigaki K, Mitomi H, Fujimori T, Ichikawa K, Tomita S Imura J, Fujii S, Itabashi M, Kameoka S, Sahara R and Takenoshita S: Immunohistochemical analysis of chromogranin A and p53 expressions in ulcerative colitis-associated neoplasia: Neuroendocrine differentiation as an early event in the colitis-neoplasia sequence. Hum Pathol 44: 2393-2399, 2013.

7. Tahara $\mathrm{T}$, Inoue $\mathrm{N}$, Hisamatsu $\mathrm{T}$, Kashiwagi $\mathrm{K}$, Takaishi $\mathrm{H}$, Kanai T, Watanabe M, Ishii $\mathrm{H}$ and Hibi T: Clinical significance of microsatellite instability in the inflamed mucosa for the prediction of colonic neoplasms in patients with ulcerative colitis. J Gastroenterol Hepatol 20: 710-715, 2005.

8. Liu X, Goldblum JR, Zhao Z, Landau M, Heald B, Pai R and Lin J: Distinct clinicohistologic features of inflammatory bowel disease-associated colorectal adenocarcinoma: In comparison with sporadic microsatellite-stable and Lynch syndrome-related colorectal adenocarcinoma. Am J Surg Pathol 36: 1228-1233, 2012

9. Baldassarre G, Belletti B, Nicoloso MS, Schiappacassi M, Vecchione A, Spessotto P, Morrione A, Canzonieri V and Colombatti A: p27(Kip1)-stathmin interaction influences sarcoma cell migration and invasion. Cancer Cell 7: 51-63, 2005.

10. Zhang HQ, Guo X, Guo SQ, Wang Q, Chen XQ, Li XN and Guo LS: STMN1 in colon cancer: Expression and prognosis in Chinese patients. Eur Rev Med Pharmacol Sci 20: 2038-2044, 2016.
11. Bruwer M, Schmid KW, Senninger N and Schurmann G: Immunohistochemical expression of P53 and oncogenes in ulcerative colitis-associated colorectal carcinoma. World J Surg 26: 390-396, 2002.

12. Watanabe T, Konishi T, Kishimoto J, Kotake K, Muto T and Sugihara K; Japanese Society for Cancer of the Colon and Rectum: Ulcerative colitis-associated colorectal cancer shows a poorer survival than sporadic colorectal cancer: A nationwide Japanese study. Inflamm Bowel Dis 17: 802-808, 2011.

13. Bhagirath D, Abrol N, Khan R, Sharma M, Seth A and Sharma A: Expression of CD147, BIGH3 and Stathmin and their potential role as diagnostic marker in patients with urothelial carcinoma of the bladder. Clin Chim Acta 413: 1641-1646, 2012.

14. Ullman TA and Itzkowitz SH: Intestinal inflammation and cancer. Gastroenterology 140: 1807-1816, 2011.

15. Carney BK and Cassimeris L: Stathmin/oncoprotein 18, a microtubule regulatory protein, is required for survival of both normal and cancer cell lines lacking the tumor suppressor, p53. Cancer Biol Ther 9: 699-709, 2010.

This work is licensed under a Creative Commons Attribution-NonCommercial-NoDerivatives 4.0 International (CC BY-NC-ND 4.0) License. 